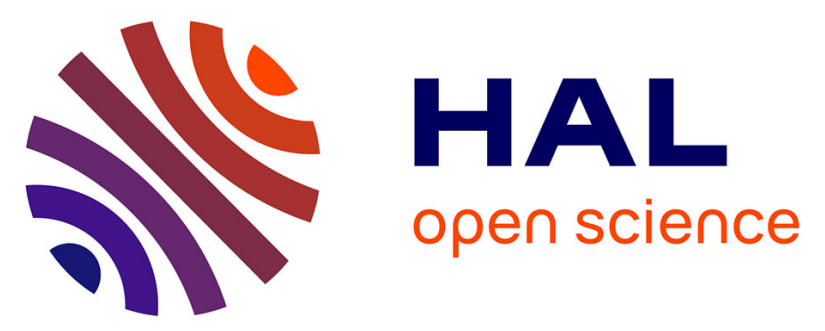

\title{
Propriétés rhéologiques et mécaniques des composites à matrice polypropylène pour l'automobile: effets couples de la pollution et du recyclage
}

Nadia Bahlouli, Cécile Rogueda-Berriet, Jacques Guillet, Claude Raveyre

\section{To cite this version:}

Nadia Bahlouli, Cécile Rogueda-Berriet, Jacques Guillet, Claude Raveyre. Propriétés rhéologiques et mécaniques des composites à matrice polypropylène pour l'automobile: effets couples de la pollution et du recyclage. Revue des composites et des matériaux avancés $=$ Journal of Composite and Advanced Materials, 2011, 20 (3/2010), 10.3166/rcma.20.373-390 . hal-00662584

\section{HAL Id: hal-00662584 \\ https://hal.science/hal-00662584}

Submitted on 25 Nov 2021

HAL is a multi-disciplinary open access archive for the deposit and dissemination of scientific research documents, whether they are published or not. The documents may come from teaching and research institutions in France or abroad, or from public or private research centers.
L'archive ouverte pluridisciplinaire HAL, est destinée au dépôt et à la diffusion de documents scientifiques de niveau recherche, publiés ou non, émanant des établissements d'enseignement et de recherche français ou étrangers, des laboratoires publics ou privés.

\section{(ㅇ)(1) $\$$}

Distributed under a Creative Commons Attribution - NonCommerciall 4.0 International 


\title{
Propriétés rhéologiques et mécaniques des composites à matrice polypropylène pour l'automobile
}

\section{Effets couplés de la pollution et du recyclage}

\author{
Nadia Bahlouli ${ }^{1}$ - Cécile Rogueda-Berriet ${ }^{2}$ \\ Jacques Guillet $^{3}$ - Claude Raveyre ${ }^{3}$
}

1. Institut de Mécanique des Fluides et des Solides

Université de Strasbourg, CNRS, 2 rue Boussingault F-67000 Strasbourg

nadia.bahlouli@unistra.fr

2. Institut FEMTO-ST, UMR 6174, Département mécanique appliquée

Université de Franche-Comté, 32 av. de l'Observatoire F-25044 Besançon cedex

3. Laboratoire de Rhéologie des Matières Plastiques (LRMP)

Univ de St-Etienne - UMR 5223 Ingénierie des Matériaux Polymères

23 rue du docteur Paul Michelon F-42023 Saint-Etienne

RÉSUMÉ. Notre étude s'intéresse au recyclage de matériaux composites à base de polypropylène majoritairement présents dans les véhicules automobiles. Les matériaux issus des véhicules en fin de vie étant pollués, nous avons choisi d'incorporer des contaminants comme l'huile moteur avant de recycler nos matériaux. Ces effets ont été identifiés non seulement à partir de la mesure du poids moléculaire et des propriétés rhéologiques mais aussi en analysant les résultats obtenus lors de la détermination des caractéristiques mécaniques. Le polluant modèle (l'huile moteur) a été incorporé dans les composites au cours du process d'extrusion. Le recyclage a été simulé en effectuant plusieurs cycles d'extrusion successifs.

ABSTRACT. The aim of this work is to study the recycling of material base PP majoritary present in cars. The materials coming from old cars are polluted. Thus, in this paper, we study contaminated and recycled materials in order to highlight the mechanisms which play an important role during the treatment of recycled materials. The effects of contamination and recycling have been identified by the way of molecular weight, rheological properties, and mechanical properties. The contaminant chosen for this study is motor oil. Recycling has been performed by successive reprocessing of contaminated materials.

MOTS-CLÉS : recyclage, extrusion, pollution, pares chocs, rhéologique, propriétés mécaniques, comportement dynamique.

KEYWORDS: recycling, reprocessing, pollution, car bumpers, rheological, mechanical properties, dynamic behaviour. 


\section{Introduction}

La forte croissance de l'utilisation des polymères dans l'industrie automobile, particulièrement pour les pièces de semi-structures, a entraîné une augmentation conséquente des déchets plastiques. Les dernières directives internationales (Directive, 2003) préconisent que la majeure partie devra faire l'objet d'une valorisation. Dans ce cadre, il est souhaitable, pour atteindre une valorisation matière des plastiques économiquement viable, que cette réutilisation se fasse dans les pièces de structure des véhicules de demain. Parmi ces déchets plastiques, une large part est composée de thermoplastiques et plus particulièrement de polypropylènes chargés (PP) et leurs dérivés copolymères qui sont largement majoritaires (environ $15 \mathrm{Kg}$ dans les dernières versions de la Mégane par exemple). C'est dans ce cadre que nous avons formé un consortium entre différents laboratoires de recherches regroupant différentes compétences pour répondre à ces appels.

Après une courte synthèse bibliographique mettant en évidence le manque de résultats sur le comportement mécanique des polymères thermoplastiques pollués et recyclés, nous présentons les matériaux utilisés dans cette étude. Ensuite nous décrivons les essais mis en œuvre pour caractériser le comportement mécanique et les mécanismes de déformation. Puis nous mettons en évidence l'intérêt de la pluridisciplinarité des partenaires du projet lors de l'analyse et de l'interprétation de nos résultats pour décrire les mécanismes mis en jeu lors du recyclage et de la pollution des matériaux polymères.

Les métaux sont facilement recyclables. Malheureusement, à propriétés mécaniques équivalentes, ce n'est pas le cas des matériaux à base polymères. Notre étude s'intéresse au recyclage isofonction de composites à base polypropylène majoritairement présents dans les véhicules. Au cours de leur utilisation, les matériaux des véhicules sont mis en présence de divers types de contaminations. Dans notre étude globale, le choix des contaminants s'est porté sur l'huile moteur (HM) et l'éthylène glycol (EG) utilisé, par exemple, dans la composition du liquide de frein. Ils ont été choisis parce que leur présence a été déterminée dans les pièces de véhicules à la fin de la durée de vie des véhicules (Directive, 2003). Les effets des contaminants sur ces deux composites à base de polymères ont été peu étudiés. (Kallel et al., 2003). Certaines études ont indiqué que l'huile ou l'éthylène glycol sont introduits dans des mélanges de polymères et composites comme comptabilisant (Othman et al., Pessey et al., 2010). Dans ce cas, certaines améliorations sont attendues sur les propriétés mécaniques des matériaux pollués. Par exemple, (Kallel et al., 2003 ; Alexander et al., 2006 ; Othman et al., 2006) ont montré que l'huile utilisée comme comptabilisant améliore la résistance à la traction du mélange des composites ou des mélanges de polymères, tandis que le module d'Young diminue. Des travaux plus complets ont été récemment présentés dans Pessey et al., (2010).

Dans la littérature, il existe plusieurs travaux traitant du retraitement des thermoplastiques purs. Certains d'entre eux concernent le recyclage du polypropylène seul. Ils signalent que cinq cycles de recyclage sont nécessaires avant 
d'observer une dégradation non négligeable des propriétés mécaniques (D’Orazio et al., 2001 ; Guerrica-Echevarria et al., 1996). Ils montrent également que la limite d'élasticité et le module d'Young augmentent jusqu'au cinquième cycle de retraitement avant d'atteindre un maximum. Ils attribuent cette amélioration des propriétés au taux de cristallisation qui augmente également avec le recyclage. Mais d'autres auteurs ont plutôt mis en évidence une dégradation des propriétés mécaniques et rhéologiques du polypropylène lors d'extrusions multiples (Aurrekoetxea et al., 2001; Guerrica et al., 1996). La caractérisation de la masse molaire ainsi que des propriétés rhéologiques et mécaniques des polymères recyclés ont donné une bonne indication sur le nombre maximal de retraitements possibles à réaliser tout en conservant un bon niveau de qualité requis pour une application spécifique. La bibliographie démontre néanmoins que la contrainte à la rupture et l'énergie de rupture ont été considérablement réduits par le processus de recyclage. En accord avec (Guerrica et al., 1996) et (Aurrekoetxea et al., 2001) l'absence de changements dans la structure chimique et l'augmentation de l'indice de fluidité de la phase PP suggèrent que la scission des chaines est le principal mécanisme de dégradation de ce type de recyclage. Avec les coupures de chaînes occasionnées par des niveaux élevés de cisaillement lors du passage successif dans l'extrudeuse, la ductilité du PP est alors réduite.

Notre étude concerne davantage des matériaux modèles représentatifs des polypropylènes issus de la filière de recyclage des véhicules en fin d'usage (VHU). La grande majorité de ces matériaux sont des polypropylènes chocs. Or, les effets du recyclage sur les matériaux à base de polypropylène issus d'un pare-choc n'ont été que partiellement étudiés (Luda et al., 2002 ; 2003). Certaines dégradations induites par le vieillissement et le recyclage des composites à base de polypropylène ont été quantifiées sous chargement quasi statique. Plus proches de nos matériaux modèles, les effets sur la dégradation des caractéristiques rhéologiques et les propriétés mécaniques des mélanges multi-extrudés de PP choc à base de poly (éthylène-coacétate de vinyle) ont également été étudiés. (Luda et al., 2002 ; Da Costa et al., 2007) ont montré que les caractéristiques rhéologiques et les propriétés physiques sont dégradées après le recyclage. Comme pour le PP seul, ils ont observé une augmentation de l'indice de fluidité à chaud (MFI) et du degré de cristallinité $\left(\mathrm{X}_{\mathrm{c}}\right)$, avec une réduction continue de la masse molaire, et de la température de fusion $\left(\mathrm{T}_{\mathrm{m}}\right)$ lorsque le nombre d'extrusions augmente. Mais, les effets observés sur le recyclage du polypropylène pur sont probablement différents de ceux observés sur le polypropylène choc ou chargé talc en raison de la présence des particules de caoutchouc et de talc. En présence d'un polluant tel que l'huile moteur, les mécanismes doivent avoir été modifiés. Cet article porte uniquement sur les effets du couplage recyclage/pollution par l'huile moteur des composites à base polypropylène chargés de talc et de particules d'élastomères. 


\section{Etude des matériaux initiaux non recyclés}

\subsection{Caractérisation physique et microstructurale des matériaux initiaux}

Notre étude porte sur deux types de matériaux fournis par SABIC :

- le PP copolymère bloc (PP greffé EPR) de nom commercial : 108MF97 utilisé pour la peau du pare-choc avant ;

- le PP compound (PP+EPDM (mélange mécanique) + talc) de nom commercial : 7510 utilisé pour la peau du pare-choc arrière.

Des plaques d'épaisseur $3 \mathrm{~mm}$ dans lesquelles ont été découpées les éprouvettes de compression dynamique, de flexion et de fluage ainsi que des éprouvettes normalisées de traction ont été injectées. Au cours de l'étude, l'isotropie des plaques a été vérifiée pour diverses directions de chargements.

Une analyse physicochimique a permis d'identifier la composition et les propriétés thermodynamiques de ces deux matériaux (tableau 1).

\begin{tabular}{|c|c|c|c|c|c|c|}
\hline Matériaux & $\mathrm{PP}(\%)$ & Elastomère (\%) & Talc $(\%)$ & $\mathrm{Tg}\left({ }^{\circ} \mathrm{C}\right)$ & $\mathrm{T}_{\mathrm{m}}\left({ }^{\circ} \mathrm{C}\right)$ & $\mathrm{X}_{\mathrm{c}}(\%)$ \\
\hline $108 \mathrm{MF} 97$ & 78 & 22 & 0,5 & -47 & 167 & 53 \\
\hline 7510 & 68 & 20 & 12 & -59 & 165 & 55 \\
\hline
\end{tabular}

Tableau 1. Composition et caractéristiques des matériaux étudiés

Les poids moléculaires et la caractérisation rhéologique ont été déterminés comme suit : les granulés de matériaux vierges et pollués ont été dissous à $135^{\circ} \mathrm{C}$ pendant cinq heures dans le trichlorobenzène stabilisé par de l'IRGANOX 1010. La référence de l'appareil pour la détermination de la répartition de la masse molaire est le dispositif de Waters Alliance 2000. Le polypropylène chargé talc et élastomère n'a pas été analysé en raison de la présence de talc. Ces résultats ne concernent que les granulés avant moulage par injection. Les propriétés rhéologiques ont été mesurées au moyen d'un rhéomètre capillaire Rheoflixer Haake. La longueur et le diamètre du capillaire étaient de 30 et $1 \mathrm{~mm}(\mathrm{~L} / \mathrm{D}=30)$. Les valeurs de viscosité sont ensuite corrigées en utilisant seulement la procédure Rabinowitch. Les mesures ont été effectuées à $230^{\circ} \mathrm{C}$ dans une gamme de taux de cisaillement de 50, 200, 500, 1000 et $3000 \mathrm{~s}^{-1}$.

\subsection{Caractérisation mécanique}

En raison de l'utilisation de ces matériaux dans des structures de type pare-choc, des essais sur une large gamme de vitesses ont été réalisés. En ce qui concerne la caractérisation pour des essais à faibles vitesses, des essais de traction et de flexion 
quasi statiques ainsi que des essais de fluage ont été réalisés. Pour caractériser le comportement dynamique, des essais de compression dynamique sur barre d'Hopkinson ont été réalisés.

Les essais de traction uniaxiaux en quasi statique à température ambiante ont été réalisés pour caractériser les propriétés mécaniques et les effets de la vitesse de déformation. Ces essais ont été réalisés sur une machine hydraulique INSTRON série 8 500. Un extensomètre INSTRON de référence 2360-102 de longueur initiale entre couteaux de $10 \mathrm{~mm}$ a été utilisé pour mesurer l'allongement des échantillons en cours d'essai. Trois paramètres ont été évalués avec ces essais : le module élastique $(E)$, la contrainte seuil $\left(\sigma_{\mathrm{y}}\right)$ et la déformation seuil $\left(\varepsilon_{\mathrm{y}}\right)$. Sur aucun de nos échantillons, il n'a été observé de phénomène de striction. La contrainte et la déformation vraie ont été calculées classiquement (Pessey et al., 2007 ; Bahlouli et al., 2009).

Les résultats présentés dans cette section ont été identifiés sur 5 essais à une vitesse de déformation constante de $10^{-3} \mathrm{~s}^{-1}$ et à température ambiante. Des essais de flexion 3 points ont été réalisés à température ambiante à l'aide d'un montage adapté à une machine de traction électrique INSTRON 6025. Les éprouvettes sont usinées directement dans la plaque injectée avec les matériaux initiaux et ont une forme de barreau de $3 * 20 * 120 \mathrm{~mm}^{3}$. Un capteur LVDT de course maximale $12,5 \mathrm{~mm}$ (précision $1 \mu \mathrm{m}$ ) est placé au centre du dispositif. Il permet l'asservissement par la flèche à une vitesse de $0,2 \mathrm{~mm} \cdot \mathrm{s}^{-1}$. En utilisant l'équivalence classique de la résistance des matériaux et compte tenu des dimensions des éprouvettes, cette vitesse équivaut à une vitesse de déformation de $2,510^{-4} \mathrm{~s}^{-1}$. D'autres essais ont également été réalisés à une vitesse de déformation de $2,510^{-3} \mathrm{~s}^{-1}$. On présente ici la courbe maîtresse de 3 essais réalisés pour chaque matériau, représentant la force mesurée en fonction de la flèche à $2,510^{-4} \mathrm{~s}^{-1}$.

Les essais de fluage en traction ont également été réalisés à température ambiante et sur des éprouvettes de traction d'aire spécifique $3 \times 10 \times 50 \mathrm{~mm}^{3}$, usinées à partir des plaques de départ, perpendiculairement au sens d'injection. La contrainte est obtenue à l'aide de poids placés à une extrémité de l'éprouvette, l'autre étant maintenue dans un portique rigide. Les essais ont été réalisés à 2, 4 et $6 \mathrm{MPa}$. Toutes ces valeurs permettent au comportement viscoélastique de rester dans le domaine linéaire. La contrainte de fluage est maintenue pendant 2 jours puis la charge est retirée, permettant la recouvrance de l'échantillon, étudiée durant 3 jours. La déformation axiale est mesurée au cours de l'essai par extensométrie mécanique. La décharge met en évidence la présence d'une déformation résiduelle. On propose ici l'évolution temporelle de la compliance, définie par :

$$
J(t)=\varepsilon(t) / \sigma_{0}
$$

$\mathrm{J}(\mathrm{t})$ est la somme d'une compliance élastique, une viscoélastique et enfin une viscoplastique. 


$$
J(t)=J_{\text {élastique }}+J_{\text {viscoplastique }}(t)+J_{\text {visco-elastique }}(t)
$$

Les essais en compression dynamique ont été réalisés sur une barre d'Hopkinson développée à l'Institut de mécanique des fluides et des solides à Strasbourg (IMFS). Ces essais ont été menés à température ambiante. L'épaisseur des échantillons est de $3 \mathrm{~mm}$ et le diamètre est de $16 \mathrm{~mm}$ en accord avec les prérogatives décrites dans (Malinowski et al., 1986). Avant chaque essai, une très fine couche de lubrifiant a été déposée sur chaque éprouvette afin de minimiser les effets du frottement. Le système de barres d'Hopkinson utilisé est composé de deux barres, une barre entrante et une barre sortante, en inox de $3 \mathrm{~m}$ de long et de $22 \mathrm{~mm}$ de diamètre. L'échantillon est positionné entre les deux barres. Un projectile de $22 \mathrm{~mm}$ de diamètre et de $1 \mathrm{~m}$ de longueur a été utilisé pour générer les ondes incidentes. Le principe est le suivant : quand le spécimen est soumis au choc, une partie de l'onde incidente se trouve transmise dans la barre sortante et l'autre partie se trouve réfléchie dans la barre entrante. Les ondes incidentes, transmises et réfléchies sont mesurées à l'aide de jauges collées sur les deux barres. Ces signaux sont ensuite utilisés pour calculer la contrainte et la déformation nominale dans l'échantillon déformé de façon homogène. Les courbes de contraintes et déformations vraies ont été obtenues selon les calculs décrits dans (Pessey et al., 2008).

\subsection{Résultats expérimentaux}

Les figures 1 et 2 représentent le comportement macroscopique obtenu lors des essais de traction uniaxiale quasi statique pour chacun des matériaux. De façon générale, les matériaux présentent la réponse mécanique d'un polymère thermoplastique soumis à un chargement quasi statique. Après une réponse élastique linéaire, une petite zone de viscoélasticité apparaît avant d'atteindre la contrainte seuil. Après ce point, le matériau se déforme plastiquement. La viscoplasticité de ce matériau peut être décomposée en deux phases: un premier domaine d'adoucissement initial plus ou moins prononcé suivi par un écrouissage. D'un autre côté, la zone utile de tous nos échantillons présente un blanchiment qui intervient très tôt dans l'histoire du chargement. Ce blanchiment correspond au phénomène de cavitation qui se déclenche dans les composites chargés de particules d'élastomères (Pessey et al., 2010). Une compétition forte entre cavitation et décohésion matrice/talc pour le matériau chargé talc apparaît. Une différence entre les courbes s'observe au niveau de la contrainte seuil. Le «coude» est plus accentué pour les matériaux chargés de talc. En effet, la littérature à ce sujet montre que les particules de talc ont tendance à augmenter le coude. Mais les différentes phases de nos matériaux n'étant pas identiques, nous ne conclurons pas sur cet effet autrement qu'en le comparant aux données de la littérature. En ce qui concerne les effets de vitesses, le module d'Young et la contrainte seuil augmentent avec la vitesse de chargement comme il est classique de l'observer sur ces polymères (Pessey et al., 2007). Cet effet est du même niveau pour les deux matériaux. 


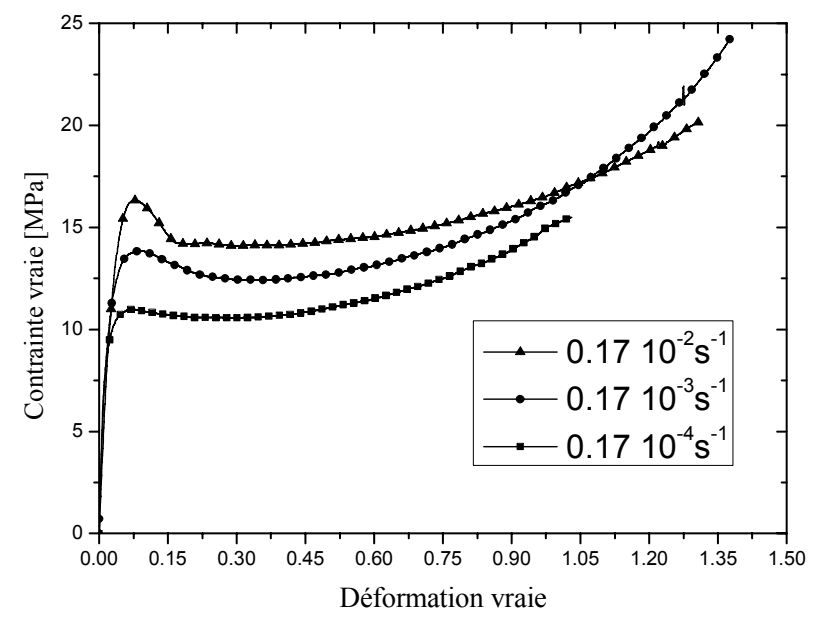

Figure 1. Courbes contrainte-déformation vraies en traction uniaxiale quasi statique à différentes vitesses de déformation du $108 \mathrm{MF} 97$

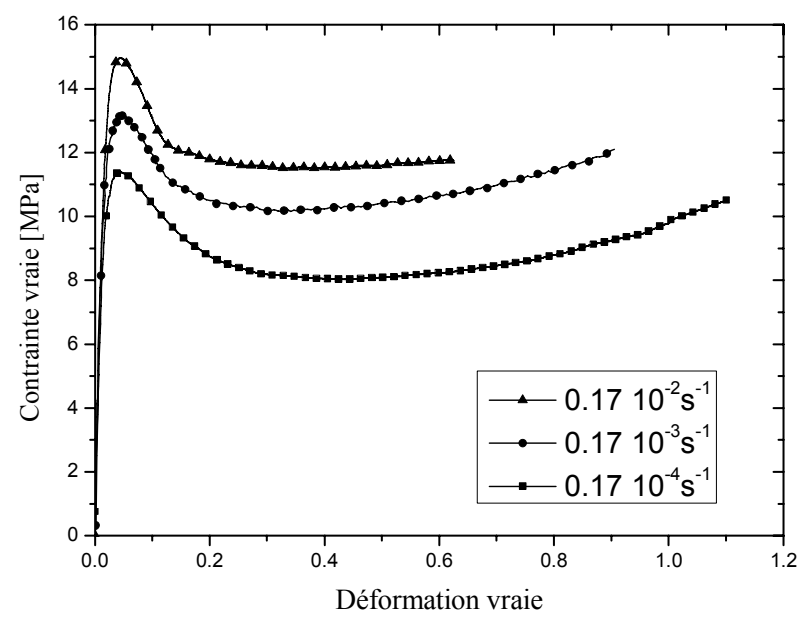

Figure 2. Courbes contrainte-déformation vraies en traction uniaxiale quasi statique à différentes vitesses de déformation du 7510

En ce qui concerne les essais de flexion (figure 3), les essais mettent en évidence un comportement totalement viscoélastique. La présence de talc dans le 7510 conduit à un comportement beaucoup plus raide et l'aire d'hystérésis est plus importante. Ces résultats sont en adéquation avec la littérature traitant des effets du 
talc sur du PP (Zhou et al., 2005). Le module de flexion initial présente, à une vitesse de déformation de $2,510^{-4} \mathrm{~s}^{-1}$, un rapport :

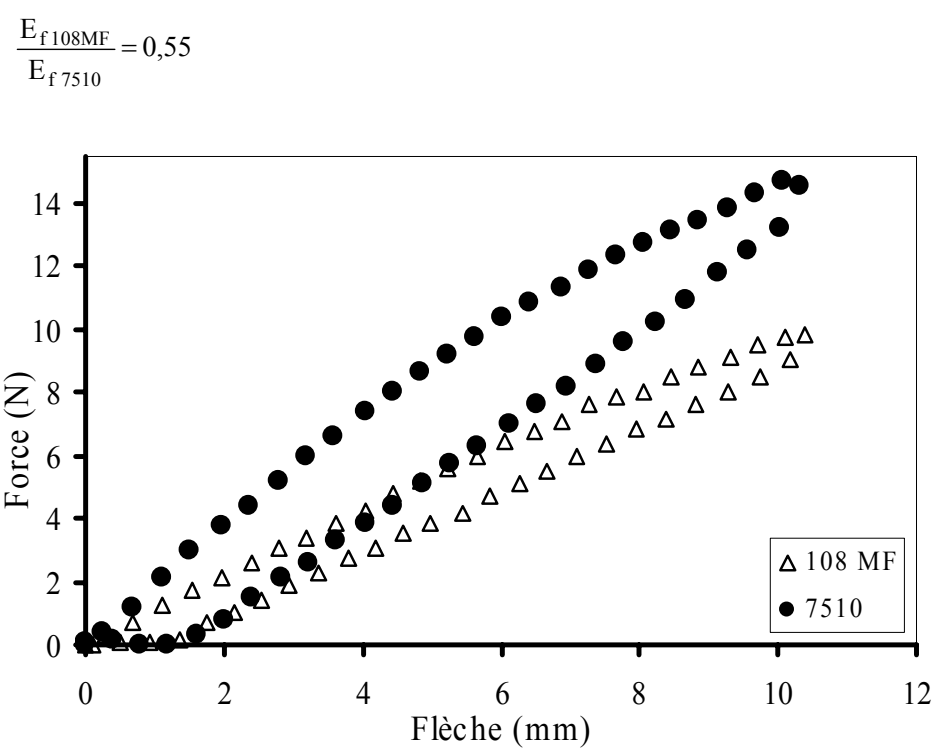

Figure 3. Courbes force-flèche en flexion à 2,5 $10^{-4} \mathrm{~s}^{-1}$ pour le 108MF97et le 7510

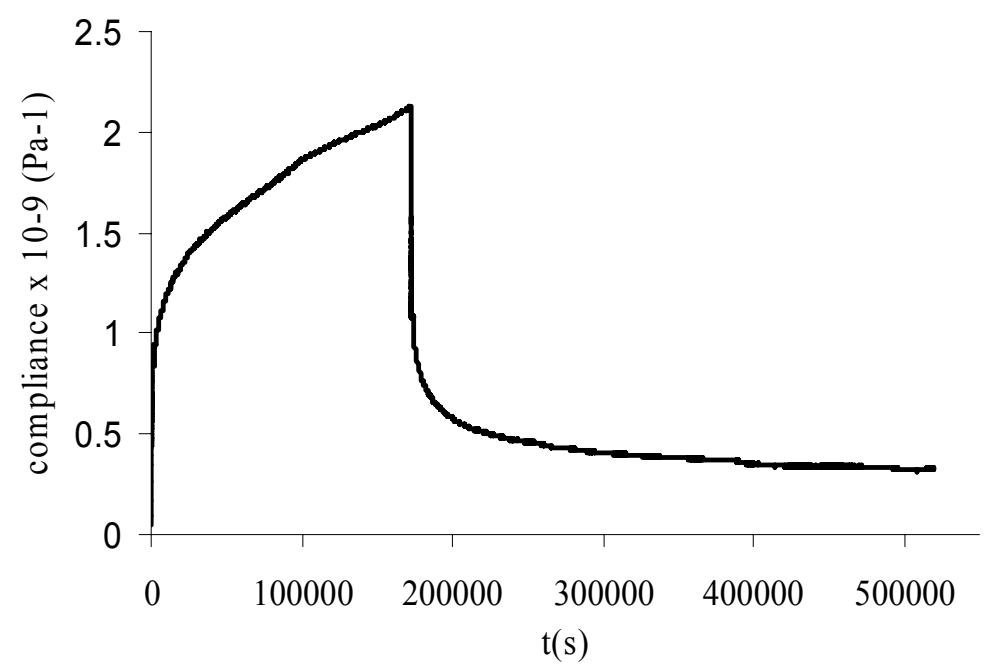

Figure 4. Courbe de compliance en fonction du temps au cours d'un essai de charge décharge pour le 7510 
Le comportement observé au cours des essais de fluage est de type viscoélastique linéaire auquel s'ajoute une composante plastique mise en évidence à la décharge (figure 4).

La présence de talc dans le 7510 lui permet d'avoir une résistance au fluage beaucoup plus importante. Le rapport des modules instantanés $\mathrm{E}_{\mathrm{I}}$ est à comparer à celui des modules de flexion :

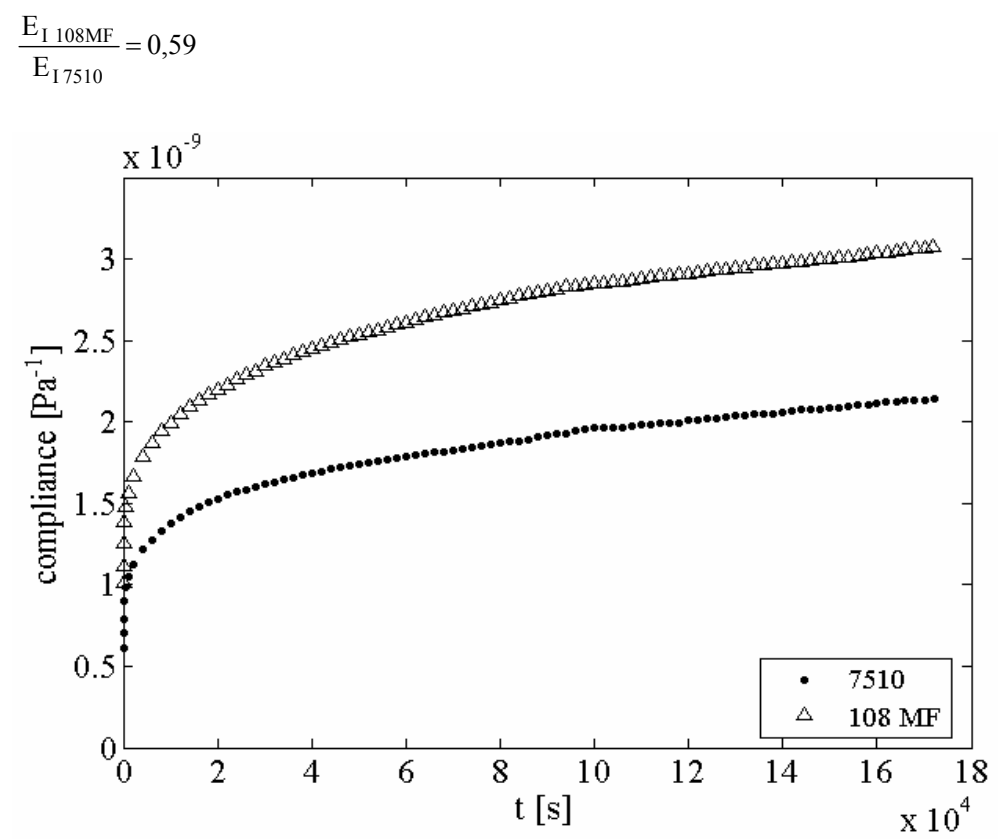

Figure 5. Courbes compliance-temps du fluage pour le 108MF97et le 7510 à $T=293^{\circ} \mathrm{C}$

Pour les essais réalisés sur la barre d'Hopkinson, le comportement dynamique obtenu pour les deux matériaux ne montre pas de contrainte seuil bien marquée (figures 6 et 7). Le comportement est de type élastoplastique linéaire.

En compression le mode de dégradation n'est pas le même, cet essai refermant les cavités. Alors qu'en traction, les matériaux cavitent fortement, en compression, seul le bord des disques montre une légère cavitation. Des observations de cryofractures au MEB ont mis en évidence de la cavitation localisée sur l'extérieur des disques. 


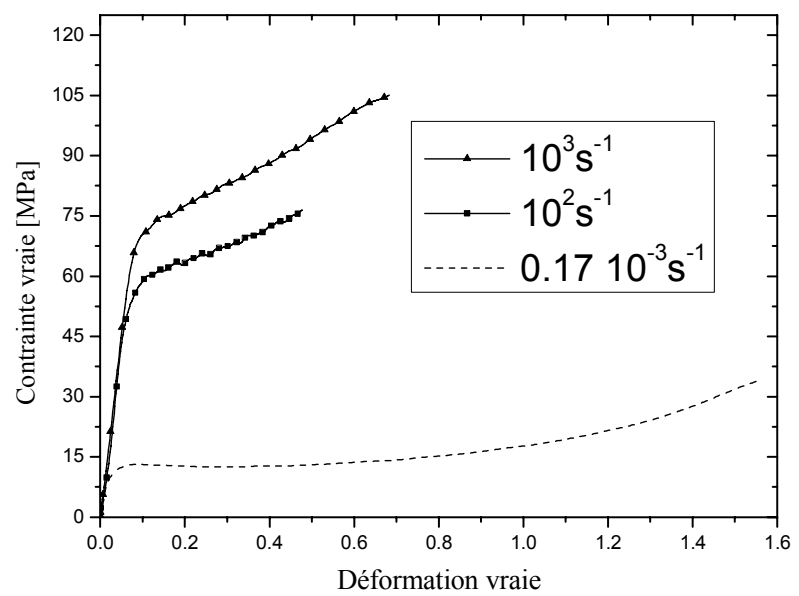

Figure 6. Courbes contrainte-déformation en compression dynamique $\grave{a} 10^{+3} s-1 \grave{a}$ $10^{+4} \mathrm{~s}^{-1}$ pour le 108MF97. La courbe de traction quasi statique à $10^{-3} \mathrm{~s}^{-1}$ est insérée à titre comparatif

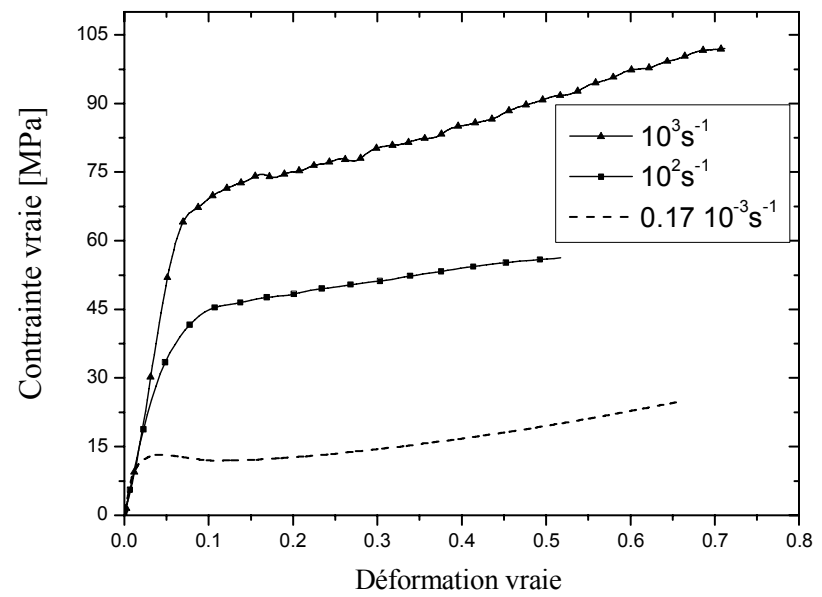

Figure 7. Courbes contrainte-déformation en compression dynamique à $10^{+3} \mathrm{~s}-1 \grave{a}$ $10^{+4} \mathrm{~s}^{-1}$ pour le 7510. La courbe de traction quasi statique à $10^{-3} \mathrm{~s}^{-1}$ est insérée à titre comparatif 


\section{Effets du recyclage et de la pollution}

\subsection{Processus de recyclage et de pollution}

Afin de reproduire l'histoire thermomécanique résultant du recyclage mécanique des pare-chocs, les granulés ont été réextrudés plusieurs fois. Nous ne présentons que les résultats pour le matériau recyclé 6 fois, ce résultat étant le plus significatif. La nomenclature choisie est décrite dans le tableau 2.

\begin{tabular}{|l|c|c|}
\hline \multicolumn{1}{|c|}{ Traitement } & 7510 & $108 \mathrm{MF97}$ \\
\hline 0 recyclage & 7510 & $108 \mathrm{MF97}$ \\
\hline 6 recyclages & $75106 \mathrm{P}$ & $108 \mathrm{MF97} 6 \mathrm{P}$ \\
\hline Pollution à l'huile moteur & $75102 \mathrm{HM}$ & $108 \mathrm{MF97} 2 \mathrm{HM}$ \\
\hline Pollution à l'huile moteur +6 recyclages & $75106 \mathrm{P} 2 \mathrm{HM}$ & $108 \mathrm{MF97} 6 \mathrm{P} 2 \mathrm{HM}$ \\
\hline
\end{tabular}

Tableau 2. Nomenclature des matériaux recyclés et pollués

Les effets de la pollution ont été reproduits par le processus suivant. Le contaminant choisi est de l'huile moteur synthétique (TOTAL ACTIVA 7000 10-40 Essence). Il a été ajouté lors du procédé d'extrusion pour simuler la pollution qui peut se produire durant le cours normal du cycle de vie des matériaux testés (Luda et $a l ., 2002)$. Les échantillons pollués à $2 \%$ d'huile de moteur seront notés NP2HM. N représente le nombre de recyclage subi par l'échantillon vierge.

Parmi les essais expérimentaux réalisés pour identifier les effets du recyclage et de la pollution, seuls sont réellement significatifs ceux obtenus en compression dynamique, en flexion quasi statique, en fluage, en résilience et en GPC.

\subsection{Effets du recyclage et de la pollution sur la masse molaire et la microstructure}

Les dégradations engendrées par le recyclage mécanique sont principalement dues au process d'extrusion. En effet, les résultats de l'étude rhéologique (Pessey et al., 2009) montrent qu'une rupture des chaînes les plus longues par le mécanisme de scission $\beta$ (masse molaire qui diminue) dégrade la phase matrice PP. L'indice de polydispersité IP diminue aussi car la probabilité de coupure des chaînes est plus importante en présence de chaînes longues et donc plus facile pour les polymères à masse molaire élevée. Ces résultats ont été confirmés par la mesure de l'évolution du MFI qui augmente avec le nombre de recyclage et par les courbes d'écoulement de la viscosité (Pessey et al., 2009). On observe aussi une diminution de la contrainte seuil sur les essais de compression dynamique qui peut être attribuée aux coupures 
de chaînes. Des analyses plus approfondies effectuées sur chacune des deux phases du 108MF97 mettent en évidence que seule la phase PP est dégradée.

\begin{tabular}{|c|c|c|c|}
\hline Référence & $\mathrm{M}_{\mathrm{n}}(\mathrm{g} / \mathrm{mol}) 10^{-3}$ & $\mathrm{M}_{\mathrm{w}}(\mathrm{g} / \mathrm{mol}) 10^{-3}$ & $\mathrm{IP}=\mathrm{M}_{\mathrm{w}} / \mathrm{M}_{\mathrm{n}}$ \\
\hline 108MF97 0P & 36 & 150 & 4.1 \\
\hline 108MF97 6P & 29 & 116 & 4 \\
\hline 108MF97 2HM & 34 & 139 & 4. \\
\hline 108MF97 6P 2HM & 23 & 129 & 5.5 \\
\hline
\end{tabular}

Tableau 3. Effets du recyclage et de la pollution sur la masse molaire, la masse moléculaire et l'indice de polydispersité pour les différentes phases du 108 MF97

Tous ces résultats sont en adéquation avec ceux de (Luda et al., 2002 ; Othman et al., 2006).

En ce qui concerne la présence du polluant, différentes conclusions peuvent être proposées. L'huile est reconnue pour avoir un rôle de comptabilisant, ce qui améliore les propriétés de l'interface dans les mélanges des composites et des composés polymères (Othman et al., 2006; Pessey et al., 2010). En effet, les molécules d'huile pénètrent à l'intérieur de la phase matrice et dans les particules d'élastomères car leur paramètre de miscibilité est proche de celui de la phase matrice et de la phase élastomère. De plus, Othman et al., (2006) ont montré le rôle stabilisant de l'huile lors de dégradations thermiques et la limitation de la réaction de coupure de chaîne du polymère dégradé en présence d'huile.

En ce qui concerne les effets couplés du recyclage et de l'huile moteur, les effets positifs de l'huile n'ont pas permis de limiter les dégradations engendrées par le processus de recyclage, les chaînes n'ont pas été « protégées » et se sont dégradées.

\subsection{Effets de la pollution et du recyclage sur les propriétés mécaniques}

Les effets de la pollution et du recyclage sont non négligeables sur les propriétés élastiques et à rupture pour différentes sollicitations quasi statiques et dynamiques.

En compression dynamique, les matériaux pollués, recyclés et pollués recyclés présentent tous la même forme de courbe de compression (figures 8 et 9).

En ce qui concerne les effets du recyclage sur les courbes de compression dynamique, les résultats sont en adéquation avec la littérature. Les auteurs cités dans l'introduction ont tous mis en évidence une diminution des propriétés mécaniques dues à la coupure des chaînes macromoléculaires. Les résultats concernaient des essais de traction et résilience. Les matériaux étudiés étant destinés à des pare-chocs, il est extrêmement utile de les tester en chargement dynamique. Les résultats en 
dynamique concordent avec ceux trouvés en traction quasi statique. La diminution des propriétés du 108MF97 provient de la diminution de la masse molaire (tableau 3). La vérification n'a pu être menée que sur le 108MF97. En effet, la présence d'un fort taux de talc dans le 7510 ne permet pas de mener l'essai par GPC. Mais il est fortement probable que dans le 7510 la phase PP voit sa masse moléculaire diminuer aussi. Le talc contenu dans le 7510 a pu se fracturer, induisant une diminution non négligeable de la taille des particules. Dans (Guerrica et al., 1996 ; Zhou et al., 2005), les effets de taille de particules mettent en évidence l'augmentation de la rigidité et la diminution de la résistance à la rupture quand la taille de particule diminue (fragilisation du matériau). Mais l'augmentation du MFI confirme l'hypothèse que la diminution de la masse molaire est la principale cause de diminution des propriétés mécaniques. Pour le 7510, l'huile moteur n'a pas un effet bénéfique probablement dû à l'absorption de l'huile par le talc.

En ce qui concerne les essais de flexion, pour le 108MF97, le recyclage semble augmenter légèrement le module. Le recyclage du matériau pollué ne modifie pas son module.

Le recyclage fait diminuer la raideur du 7510 mais l'ajout d'huile moteur sur le matériau vierge est beaucoup plus significatif sur le comportement. Cependant, le recyclage du matériau pollué conduit à un module légèrement plus élevé que le pollué d'origine. Mais la différence n'est pas suffisamment significative pour conclure sur un effet marqué de l'huile moteur sur le processus de recyclage.

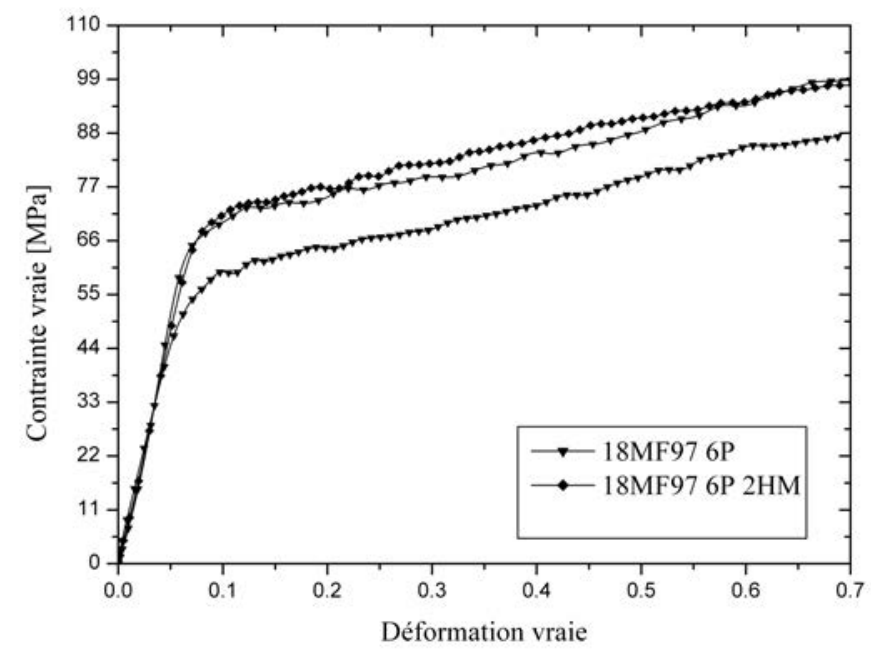

Figure 8. Courbes contrainte-déformation en compression dynamique à $10 \mathrm{~s}^{+2}$ pour les matériaux 108 MF97 recyclés et pollués 


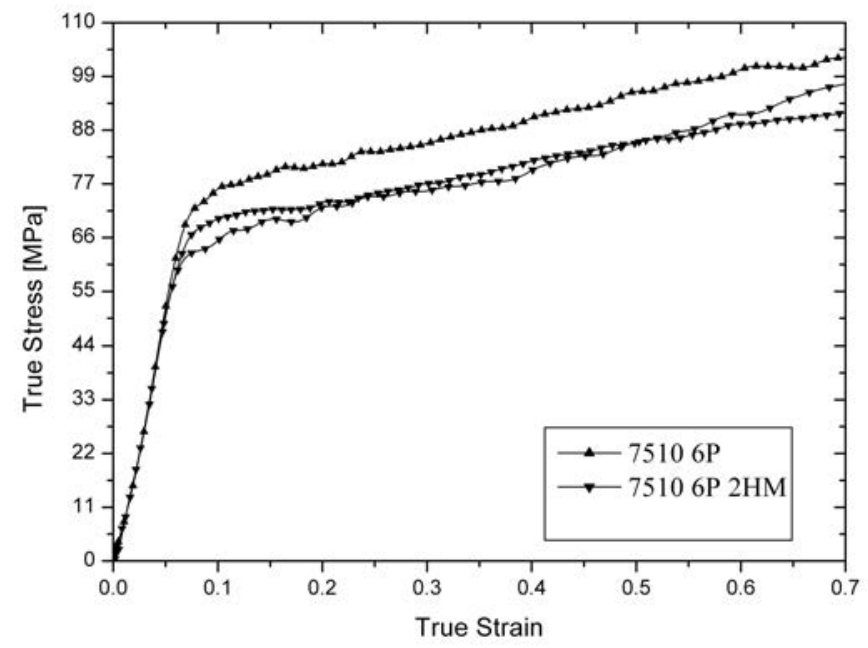

Figure 9. Courbes contrainte-déformation en compression dynamique à $10 \mathrm{~s}^{+2}$ pour les matériaux 7510 recyclés et pollués

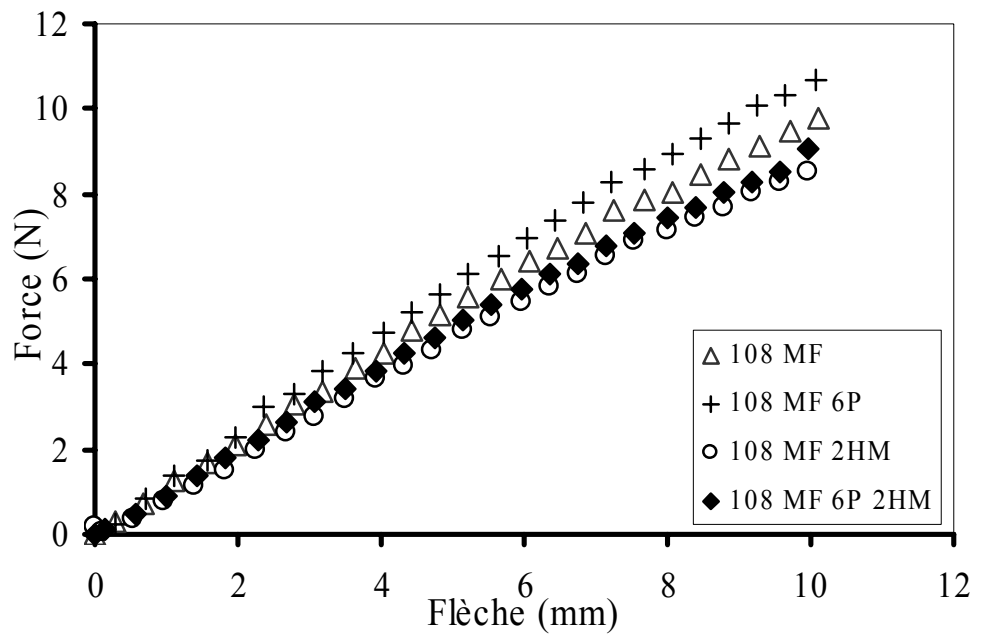

Figure 10. Courbes force en fonction de la flèche en flexion pour le 108 MF97 à 2,5 $10^{-4} \mathrm{~s}^{-1}$ : influence du recyclage et de la présence d'huile 


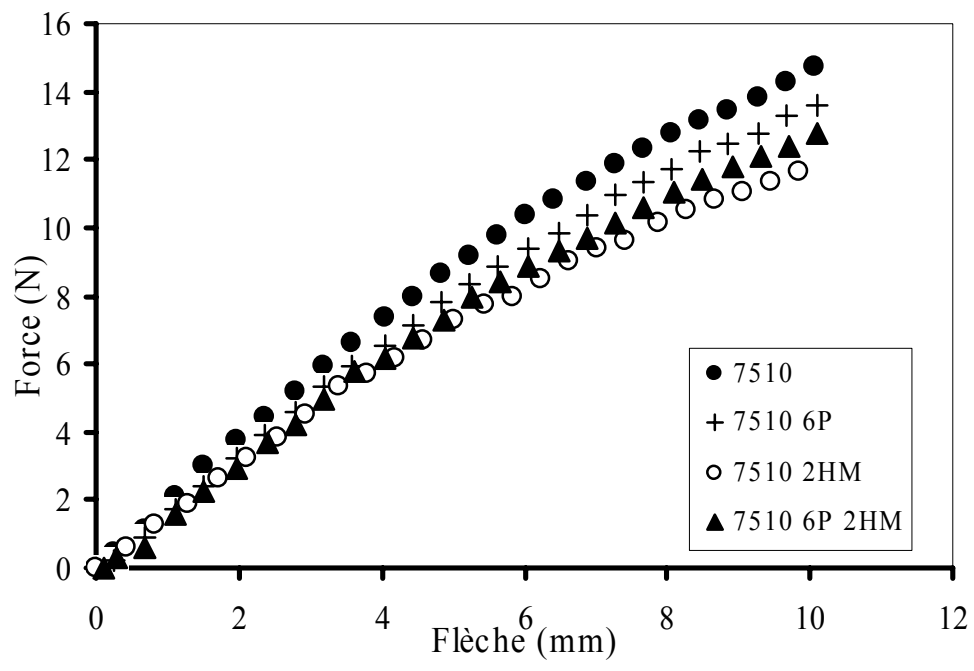

Figure 11. Courbes force en fonction de la flèche en flexion pour le 7510 à $2,510^{4}$ $s^{-1}$ : influence du recyclage et de la présence d'huile

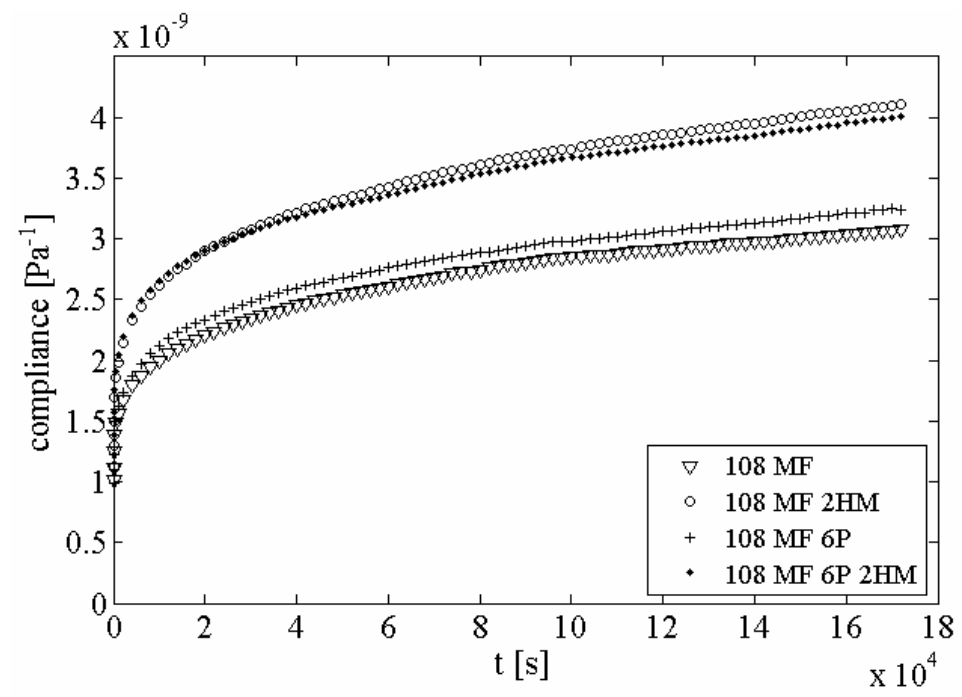

Figure 12. Courbes compliance-temps du fluage pour le 108MF97: influence du recyclage et de la présence d'huile

Le recyclage et la présence d'huile moteur font fluer beaucoup plus rapidement les deux matériaux. Cependant, les figures 12 et 13 montrent que le recyclage a une 
influence beaucoup moins importante que la présence d'huile qui, jouant son rôle de plastifiant, augmente considérablement la compliance mesurée.

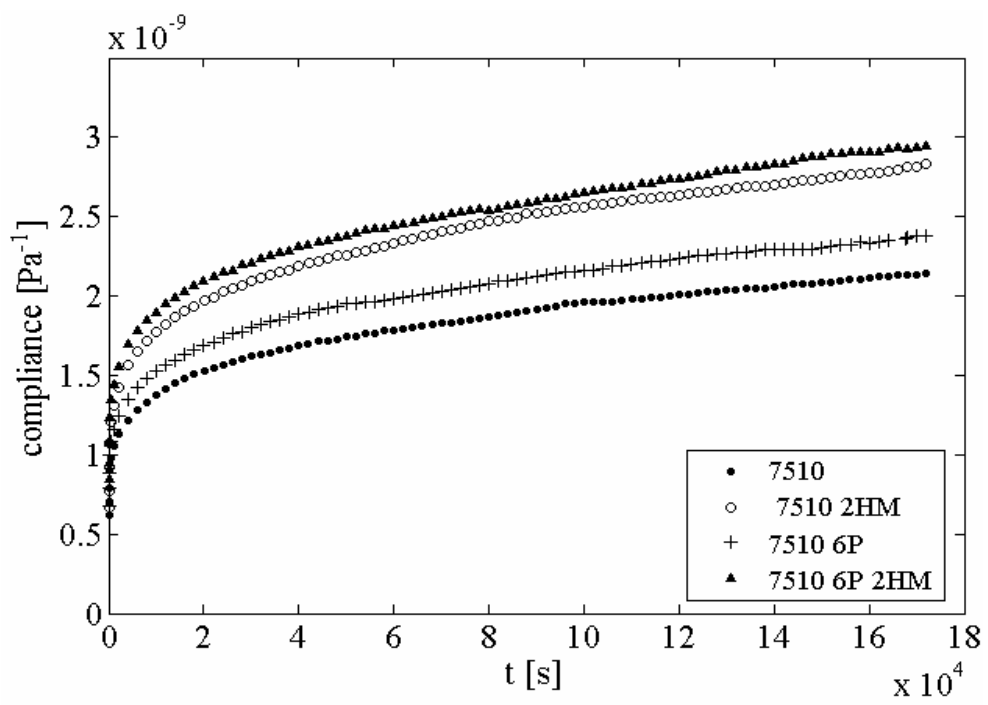

Figure 13. Courbes compliance temps du fluage pour le 7510 : influence $d u$ recyclage et de la présence d'huile

\section{Conclusion}

Cette étude expérimentale sur des matériaux vierges, pollués et recyclés a permis de mettre en évidence des résultats concernant les effets du recyclage sur des matériaux pollués. L'étude du recyclage thermomécanique conduit principalement à une détérioration des propriétés mécaniques élastiques et de la ductilité, ainsi qu'une augmentation de la cavitation. Tout ceci est principalement attribué à une diminution de la masse molaire de la phase PP généré par une scission des chaînes macromoléculaires au cours des différents cycles successifs d'extrusion.

Mais la présence d'un polluant comme l'huile moteur dans le 108MF97, le matériau sans talc, compense les effets du recyclage en agissant comme plastifiant et en limitant les dégradations occasionnées par les outils et le frottement entre chaînes à l'origine de leur scission. Par contre, la présence de particules de talc semble limiter les effets du polluant en absorbant partiellement l'huile moteur.

\section{Remerciements}

Les auteurs remercient vivement le ministère de la Recherche et de l'Innovation ainsi que le ministère de l'Enseignement Supérieur pour le financement de cette 
étude. Les auteurs remercient chaleureusement Roger Schmitt pour le précieux temps passé à préparer les échantillons, toute l'équipe de RECYPRO B (Daniel Pessey, Olivier de Almeida, Fabienne Touchard, Jean-Marc Pelletier, Catherine Gauthier et Laurence Chocinski,) ainsi que Abdessalam Dahoun et Jean-Marie Hiver pour les riches discussions au cours des trois années de ce projet.

\section{Bibliographie}

Alexander M., Thachil E.T., "A comparative study of cardanol and aromatic oil as plasticizers for carbon- black-filled natural rubber", Journal of Applied Polymer Science, vol. 102, 2006, p. 4835-4841.

Aurrekoetxea J., Sarrionanda M.A., Urrutibeascoa I., Maspoch M. LI., "Effects of recycling on the microstructure and the mechanical properties of isotactic polypropylene", Journal of Materials Science, vol. 36, 2001, p. 2607-2613.

Bahlouli N., Gauthier C., Pelletier JM, Pessey D., "Micromechanical approach of the dynamic behavior of a high impact PP and PP compound: recycling and pollution effects", Journal of Physique IV, vol. 1, 2009, p. 703-708.

Bahlouli N., Pessey D., Ahzi S., Rémond Y., "Mechanical behaviour of composite based polypropylene : recycling and strain rate effect", Journal of Physique IV, France, vol. 134, 2009, p. 1319-1323.

Da Costa H.M., Ramos V.D., Rocha M.C.G., "Rheological properties of polypropylene during multiple extrusion", Polymer. Testing, 2005, vol. 24, p. 86-93.

Da Costa H.M, Ramos V.D., De Oliveira M. G., "Degradation of polypropylene during multiple extrusions: thermal analysis, mechanical properties and analysis of variance", Polymer. Testing, vol. 26, 2007, p. 676-684.

Directive 2003/53/CE, du Parlement Européen et du Conseil, Journal officiel de 1'Union européenne, 2003.

D'orazio L., Greco R., Martuscelli E., Ragosta G., "Recycling of a plastic car component having a multilayer structure: Morphological and mechanical analysis", Polymer. Engineering and Science, vol. 36, 2001, p. 2607-2613.

Guerrica-Echevarria G, Eguiazabal J I, Nazabal J, "Effects of reprocessing conditions on the properties of unfilled and talc-filled polypropylene", Polymer degradation and stability, vol. 53, 1996, p. 1-8.

Kallel T., Massardier-Nageotte V., Jaziri M., Gérard J.F., Elleuch B, "Effect of model pollutants on the recycling of PE/PS Plastic blends", Progress in Rubber, Plastics and Recycling technology, vol. 19, ${ }^{\circ} 2,2003$, p. 61-76.

Luda M. P, Ragosta G, Musto P, Pollicino A., Caminom G. Recca A, Nepote V, "Natural ageing of automotive polymer components: characterisation of new and used poly(propylene) based car bumpers", Macromolecular Materials Engineering, vol. 287, 2002, p. 404-411. 
Luda, M.P., Ragosta, G., Musto, P, Acierno, D, Di Maio, L., Camino G., Nepote V., "Regenerative recycling of automotive polymer components: poly (propylene) based car bumpers", Macromolecular Materials Engineering, vol. 288, 2003, p. 613-411.

Maiti S, Sharma NKK, "Studies on polypropylene composites filled with talc particles. Part I: Mechanical properties", Journal of Materials Science, vol. 27, 1992, p. 4605-4613.

Malinowski JZ, Klepaczko JR, "Dynamic frictional effects as measured from the split Hopkinson bar", International Journal of Mechanical Science, vol. 28, 1986, p. 381-391.

Othman N., Ismail H., Mariatti M., "Effect of compatibilisers on mechanical and thermal roperties of bentonite filled polypropylene composites", Polymer Degradation and stability, vol. 91, 2006, p. 1761-1774.

Pessey D., Bahlouli N., Ahzi S., Khaleel M.A., "Strain Rate Effects on the Mechanical Response of Composite Based Polypropylene Deformed at Small Strains", Polymer Science-Series A, vol. 50, $\mathrm{n}^{\circ}$ 6, 2007, p.690-697.

Pessey D., Bahlouli N., Pattofato S., Ahzi S, "Polymer composites for the automotive industry: Characterization of the recycling effect on the strain rate sensitivity", International Journal of Crashworthiness, vol. 13, $\mathrm{n}^{\circ}$ 4, 2008, p. 411-424.

Pessey D., Bahlouli N., Raveyre C., Guillet J., Hiver J.M, Dahoun A., Ahzi S., "Characterization of pollution effects for two PPbased materials", Polymer Engineering and Science, vol. 50, $\mathrm{n}^{\circ} 1,2010$, p.1-9.

Zhou Y., Rangari V., Mahfuz H., Jeelani S., Mallick P.K.., "Experimental study on thermal and mechanical behavior of polypropylene talc/polypropylene and polypropylene/clay nanocomposites", Material Science and Engineering, A402, 2005, p. 109-117. 